\title{
La mort des anciens officiers de la Grande Armée à travers l'exemple charentais
}

\section{Stéphane Calvet}

\section{(2) OpenEdition \\ 1 Journals}

Édition électronique

URL : https://journals.openedition.org/ahrf/9343

DOI : $10.4000 /$ ahrf.9343

ISSN : 1952-403X

\section{Éditeur :}

Armand Colin, Société des études robespierristes

\section{Édition imprimée}

Date de publication : 1 juin 2007

Pagination : 165-183

ISSN : 0003-4436

\section{Référence électronique}

Stéphane Calvet, "La mort des anciens officiers de la Grande Armée à travers l'exemple charentais », Annales historiques de la Révolution française [En ligne], 348 | Avril-Juin 2007, mis en ligne le 01 juin 2010, consulté le 28 avril 2022. URL : http://journals.openedition.org/ahrf/9343 ; DOI : https://doi.org/ 10.4000/ahrf.9343

Ce document a été généré automatiquement le 28 avril 2022.

Tous droits réservés 


\title{
La mort des anciens officiers de la Grande Armée à travers l'exemple charentais
}

\author{
Stéphane Calvet
}

1 Le bicentenaire de l'Empire donne lieu à un florilège de rééditions de Mémoires des témoins de ce temps. Parmi eux les officiers de la Grande Armée figurent en bonne place. Les cahiers du très célèbre Coignet ${ }^{1}$ côtoient les témoignages de personnes dites importantes comme Lejeune ${ }^{2}$ et ceux de soldats plus humbles comme Levavasseur ${ }^{3}$ ou le capitaine François ${ }^{4}$. Néanmoins, cette mode éditoriale ne peut pas masquer les lacunes importantes de l'historiographie napoléonienne. En publiant ces documents majeurs sans analyse critique, on véhicule une nouvelle fois les clichés les plus simplistes qui ont prévalu tout au long du $\mathrm{XIX}^{\mathrm{e}}$ et du $\mathrm{XX}^{\mathrm{e}}$ siècle à propos des officiers de la Grande Armée. Ne serait-il pas temps de relire avec un regard neuf des textes qui, pour la plupart, ont été rédigés longtemps après les événements relatés? La question exige, en effet, de nouvelles interrogations sur les auteurs, sur ces officiers qui ont servi l'empereur entre 1800 et 1815 . Connaissons-nous aussi bien ces acteurs majeurs de l'épopée napoléonienne ? Car on oublie trop souvent que les mémorialistes militaires, à l'instar de celui qu'ils ont servi, ont voulu construire leur propre image. Une très grande majorité en effet ne reste pas neutre sur le plan politique et s'affiche parmi les nostalgiques d'un empereur qu'on admire. Outre ces prises de position, ils insistent sur les moments de gloire, leurs actions d'éclat et celles de leurs camarades, mais passent sous silence des gestes ou des attitudes moins avouables. Doit-on alors, comme ce fut souvent le cas, considérer ces hommes comme les porte-parole de tous les anciens officiers de l'Empire qui n'ont pas eu la possibilité ou la volonté de prendre une plume? Comment doit-on interpréter leurs silences ? À l'heure où notre discipline s'est enrichie des apports des autres sciences humaines telles que la sociologie ou l'ethnologie, il paraît intéressant de projeter un regard novateur sur une catégorie que l'on croit bien connaître. À ce titre, les crépuscules de vie des anciens officiers de la Grande Armée apparaissent comme des instants privilégiés où l'on peut saisir une réalité plus complexe, fort éloignée de celle construite par la légende. Par le croisement des sources 
locales et nationales, chères à l'histoire sociale et culturelle, ces hommes, porteurs d'une expérience inédite, ne correspondent pas toujours à l'image traditionnelle forgée par Balzac ${ }^{5}$. La guerre a laissé un très grand nombre de traces souvent moins reluisantes que l'éclat de la Légion d'honneur et sans doute beaucoup plus profondes. Les marches sous le soleil chaud d'Espagne ou dans la neige russe, les batailles qui font encore disserter les experts militaires napoléoniens ont bouleversé la vie des officiers bien après la fin de l'Empire. Par le biais des actes notariés, des archives de l'enregistrement, des sources privées et des documents administratifs municipaux bien trop souvent négligés par les historiens, on peut proposer une nouvelle grille d'analyse et se demander comment ces vétérans, dans les années d'après-guerre, vont tenter de s'intégrer dans une société qui, au départ, ne leur accorde guère l'attention qui leur est due. Au-delà du regard porté sur eux par leurs contemporains, on s'interrogera aussi sur leur propre système de représentation sans perdre de vue l'extrême diversité de leurs origines sociales.

2 Une enquête sur le plan national étant matériellement irréalisable, il a semblé plus percutant de l'entreprendre dans un cadre géographique restreint où il est plus facile de suivre, du berceau à la tombe, les hommes qui obtiennent l'épaulette sous l'Empire. C'est la raison pour laquelle a été choisi le département de la Charente où les sources n'ont guère été troublées par les vicissitudes du temps. Cependant cette approche régionale nécessite une rigueur méthodologique qui induit une sélection rigoureuse des documents. À cette fin ont été exploités les archives du SHD (Service Historique de la Défense) où sont conservées les revues de l'an XI (une par régiment), l'enquête de 1814, de 1816 et les registres-matricules du corps des officiers de $1814-1815^{6}$. Ces papiers qui signalent, outre les états de service, le lieu de naissance et le domicile, ont permis de constituer une première liste qu'il a fallu compléter avec l'aide des séries $\mathrm{L}$ et $\mathrm{R}$ des archives départementales de la Charente ${ }^{7}$. Assez bien tenues pour la période 1791-1815, ces deux dernières séries, riches de nombreux registres et de listes nominatives de volontaires et de conscrits, ont considérablement augmenté les effectifs du corpus initial. Par la suite, les dossiers de pension et les dossiers personnels ont systématiquement été dépouillés pour obtenir le maximum d'informations ${ }^{8}$. Loin de rechercher l'exhaustivité, cette méthode par laquelle a été établi un corpus de 507 officiers natifs de la Charente permet surtout d'apprécier les diverses raisons qui ont conduit de nombreux Charentais à obtenir l'épaulette de 1799 à 1814. Elle prend en compte aussi la très forte hétérogénéité de cette catégorie tant au plan des origines sociales que des parcours et des destins d'après-guerre.

3 S'arrêter ainsi sur leurs derniers jours permet de mieux saisir la vie de ces hommes après Waterloo. Plus qu'un bilan, cette étude a l'avantage de se placer au cœur de la question des représentations dans la France de la première moitié du XIX ${ }^{\mathrm{e}}$ siècle avec en toile de fond les vingt ans de guerre de la Révolution et de l'Empire. S'il paraît intéressant de mesurer d'abord l'impact de ces dernières sur la mobilité de ces hommes, il convient par la suite de s'attarder sur l'instant de la mort proprement dite. Observer les attitudes de ces militaires au moment suprême permet de mettre en relation leur propre expérience de la guerre, où tant de corps sont tombés à leurs côtés, avec le schéma mental de la société civile, qui développe alors le culte des morts9. Les fins de vie sont aussi primordiales pour étudier, non seulement l'image qu'ils veulent laisser d'eux-mêmes, mais aussi le regard que portent sur eux leurs contemporains. Guerres et mobilités 
4 Les guerres de l'Empire, bien plus que celles de la Révolution, ont conduit des centaines de milliers d'hommes sur les routes de France et d'Europe. En raison de la stratégie napoléonienne ou de celle de l'ennemi (Espagne), les déplacements sont réguliers et se font sur des dizaines voire des centaines de kilomètres. Lorsque sonne le glas de la Grande Armée en 1812, les mouvements s'accélèrent et deviennent permanents jusqu'à la première abdication en avril 1814. Les dossiers des officiers conservent les traces de ces campagnes et montrent que certains ont parcouru en peu de temps, souvent à pied, plus de 10000 kilomètres. Si ces migrations militaires ont sans aucun doute induit de nouveaux comportements pour les officiers, on peut s'interroger néanmoins sur leur réel impact et mesurer le déracinement provoqué par la guerre.

Sur les 507 officiers relevés, les états de service signalent que 85 trouvent la mort sous l'Empire tandis que 15 sont portés disparus en Russie ou dans les colonies. Parmi les 407 survivants, 78 n'ont pas laissé de trace de leur décès dans les archives actuellement consultables. Ils sont en revanche 329 dont le décès est mentionné dans les dossiers de pension, les tables de succession ou l'état-civil. Si 87 décèdent dans un autre département ou à l'étranger, 242 finissent leurs jours en Charente soit les 3/4 des rescapés des guerres napoléoniennes. Ce sont en très grande partie des militaires ayant obtenu une retraite à la fin de l'Empire ou durant les premières années de la Restauration (77\%), les autres étant simplement des démissionnaires et des réformés de l'armée qui reprennent l'activité professionnelle qu'ils quittèrent au moment de leur appel. Si $42 \%$ vivent leurs derniers jours dans leur commune natale, $16 \%$ s'établissent dans une commune de leur canton de naissance. Si l'on tient compte de la distance séparant la commune de décès de la commune de naissance, ils sont en réalité $68 \%$ à avoir fait moins de dix kilomètres. Cela veut dire qu'un peu plus d'un tiers a accepté un éloignement assez conséquent, le quart ayant élu domicile dans un autre département. Ces derniers sont essentiellement des officiers qui ont continué à servir sous la Restauration et la Monarchie de Juillet ou le Second Empire. Ils élisent domicile en fonction du régiment qu'ils servent et prennent souvent leur retraite dans une ville où le séjour, assez long, a permis de nouer des relations intéressantes pour un mariage. Même si ce dernier est déterminant pour la désignation du domicile final, il faut qu'il corresponde aussi avec la fin du service actif. Les officiers se marient très tardivement et attendent souvent un poste fixe ${ }^{10}$. Ainsi le lieu de décès de ces officiers correspond souvent à la géographie militaire de la France de cette première moitié du XIX ${ }^{e}$ siècle. Les $2 / 3$ décèdent effectivement à Paris, dans les villes de l'Est et les places fortes du littoral. Mais un tiers a élu domicile au moment de sa mort dans une commune d'un département limitrophe. Ce sont souvent des gendarmes qui, tout au long de leur carrière, ont tenté de se rapprocher, petit à petit, de leur département d'origine, preuve que le déracinement n'est jamais accepté totalement même pour des officiers de métier qui ont passé une partie de leur vie sur les routes de France et d'Europe.

Cependant ces derniers ne sont pas les seuls à avoir accepté une vie itinérante. Il existe, en effet, une catégorie très particulière constituée d'officiers qui n'ont pas eu la chance d'obtenir une retraite ou qui ont souffert de la modestie de la pension attribuée par le gouvernement. N'ayant aucune attache particulière dans le département et disposant $\mathrm{du}$ très maigre héritage laissé par leurs parents, ils osent se lancer dans diverses activités industrieuses après avoir subi un premier échec au chef-lieu de leur département. Tel est le cas du capitaine Jean Saint-Loup, originaire du village de Chatignac, qui après vingt-cinq ans de campagne et un mariage en Italie avec une 
Française de Valenciennes, s'installe à Baignes (en Charente), chef-lieu de canton de sa commune natale ${ }^{11}$. Après la naissance de ses premiers enfants, il élit domicile à Angoulême où il réside de 1822 à $1836^{12}$. Puis en janvier 1837, il quitte, avec toute sa famille, le chef-lieu de la Charente pour Bordeaux où il désire faire du commerce, ses premières entreprises n'ayant guère été fructueuses à Angoulême ${ }^{13}$. Il décède finalement à Valenciennes dans le Nord en 1840, c'est-à-dire dans la ville natale de son épouse qui conserve à cette date quelques biens laissés par ses parents ${ }^{14}$.

7 Il existe toutefois des officiers qui changent de domicile pour des raisons parfois difficiles à déterminer. Les documents sont effectivement très silencieux sur les motivations qui ont conduit le capitaine Jean Piaud, amputé d'un bras après la bataille de Wagram, à quitter la ville d'Angoulême alors qu'il était âgé de plus de 60 ans. Signalé comme capitaine adjudant-major de la garde-nationale, recommandé par le maire d'Angoulême et par le préfet, apprécié par la plupart de ses concitoyens, il réside en 1835 avec sa famille à Bergy dans l'Ain avant de décéder, seul, dans la petite commune d'Irigny, dans le Rhône, le 18 septembre $1844^{15}$. Sa succession indique que son épouse réside à cette même date à Ferney-Voltaire dans l'Ain et que ses deux fils sont négociants, l'un à Tours et l'autre à Lyon $^{16}$. Comment expliquer la dispersion géographique de cette famille? Pour quelles raisons un vieil infirme qui dispose encore de biens mobiliers à Angoulême, lors de son décès, a-t-il accepté de nouveau ce déracinement? De tels exemples sont nombreux et représentent environ le 1/4 des officiers morts dans un autre département, proportion qu'il convient sans doute de revoir à la hausse en intégrant une partie du contingent des vétérans dont le décès n'a pas été retrouvé. En dehors du fait qu'ils ont accepté de quitter leur région natale, ces hommes ne présentent guère de points communs et ne correspondent pas à un officier type. On retrouve parmi eux des nobles, des fils de notables mais aussi des fils de cultivateurs ou d'artisans. Les fortunes sont aussi variées que les origines sociales et cet élément ne peut pas être la seule explication de leur mobilité. Il s'agit sans doute d'une combinaison de plusieurs facteurs mais il est certain que pour les officiers subalternes montés en grade à la fin de l'Empire, l'aide parcimonieuse de l'État, le faible héritage familial et la disparition des liens sociaux traditionnels ont été déterminants. Ils sont ainsi, avec d'autres catégories professionnelles comme les ouvriers, les acteurs majeurs de ces flux migratoires qui affectent de plus en plus la France du XIX ${ }^{\mathrm{e}}$ siècle.

Parmi eux, il existe néanmoins des « expatriés » qui, au soir de leur vie, reviennent se fixer dans la commune de leur enfance comme si pour certains il était important de finir ses jours à l'ombre du clocher qui les avait vu grandir. En témoigne la femme du général Laroche qui demande au préfet de la Charente, en 1822, de respecter le vœu de son mari, exilé dans le département de l'Oise, qui a « exprimé le souhait de mourir sur sa terre natale $\aleph^{17}$. Si effectivement $75 \%$ des officiers environ décèdent en Charente, ils sont nombreux cependant à avoir séjourné au moins une fois dans de grandes villes françaises. La lecture de la correspondance du maire d'Angoulême de 1815 à 1860 permet de reconstituer l'itinéraire de nombreux vétérans avant que ceux-ci ne décident, pour diverses raisons, de finir leurs jours à Angoulême. Mort en 1872 à Angoulême dans la maison de son gendre, le lieutenant retraité Jean-Noël Bouniceau a mené une vie particulièrement itinérante depuis l'obtention de sa pension en 1812 jusqu'à sa mort. Alors qu'on le retrouve domicilié à Angoulême pour la naissance de ses cinq premiers enfants sous la Restauration, on perd sa trace peu avant la Révolution de juillet 1830. À cette date, il est séparé de corps et de biens d'avec son épouse qui ne supporte plus le goût trop prononcé de son mari pour le jeu, s'il faut en croire les 
rapports du commissaire de police d'Angoulême. Criblé de dettes, cet officier, qui arbore toujours fièrement sa décoration de la Légion d'honneur, y compris lorsqu'il joue dans les tavernes clandestines, quitte Angoulême. Son itinéraire a été soigneusement reconstitué par l'intermédiaire de lettres dans lesquelles se plaignent les aubergistes, les restaurateurs, les maitres de pension et les libraires qui ont le malheur de faire confiance à ce "digne légionnaire». Huit lettres ont ainsi été adressées au maire d'Angoulême entre 1831 et 1837. On reproche au « Sieur Bouniceau originaire d'Angoulême et ancien officier » de ne pas avoir payé les notes de frais. On apprend ainsi qu'il fait escale à Bordeaux puis à Nantes et enfin à Paris, où il séjourne dans différents établissements. Sa présence est attestée en Charente en 1838 puisqu'il vient pleurer sur la tombe de ses filles en acceptant de contribuer au paiement d'une concession perpétuelle. Il déménage ensuite plusieurs fois sans quitter le département ${ }^{18}$. Ne possédant rien, il finit ses jours chez sa fille qui, après son mariage, accepte de le prendre en pension. Dans cet exemple on peut voir que le retour dans la ville natale est conditionné autant par la détresse financière que par la bienveillance familiale ${ }^{19}$.

9 Mais la terre natale semble être parfois la dernière attache sentimentale de l'officier solitaire et ruiné. Comment expliquer alors le « retour au foyer » du chef de bataillon Jean Thouars qui meurt à l'hospice d'Angoulême en janvier 1830 veuf et sans enfant? Sa carrière militaire l'arrache à sa ville natale en 1778. Alors qu'il est en Amérique entre 1779 et 1783, on le retrouve marié et domicilié à Paris pendant la Révolution où il profite des événements pour acquérir des biens nationaux. Après de nombreuses péripéties militaires qui l'obligent à suivre la Grande Armée en 1806 et 1807, il est de retour dans la capitale en 1809 à cause de son «ivrognerie " répétée et de son « incompétence militaire » qui lui valent d'être emprisonné plusieurs fois et qui ont finalement raison de son ascension sociale. Sa malhonnêteté et sa rancune vis-à-vis des autorités militaires scellent le destin de cet homme qui, "sans ressources et malade ", revient à Angoulême vers 1825 pour y mourir cinq ans plus tard avec pour seul réconfort celui des employés de l'hospice de Beaulieu ${ }^{20}$. Ces retours au pays, au crépuscule de la vie, sont difficiles à quantifier mais leur existence montre que l'attachement à la terre des ancêtres se maintient malgré la guerre et les expériences de chacun.

10 Comment expliquer alors la "sédentarisation » de ceux qui ont choisi de "rentrer au pays " dès l'obtention d'une retraite? L'exploitation de sources privées, chaleureusement mises à notre disposition, montre que de nombreux militaires sont intégrés dans des réseaux de parentèle extrêmement forts qui ne leur laissent guère le choix de poursuivre une vie dans un autre " pays $"^{21}$. Dès 1804 , le jeune sous-lieutenant Brothier qui a suivi le général Bonaparte en Italie et en Égypte, se marie avec Élisabeth Pelletier, lointaine cousine du côté paternel. Une enquête approfondie, menée autour des personnages cités dans les cahiers de son fils, montre que tous, y compris les compagnons d'armes issus du même régiment et natifs de la même commune, sont liés à la famille Brothier depuis des générations. Ses parents, vivants en 1804, ont préparé le mariage qui fixe définitivement cet ancien officier retraité, devenu épicier sur la place du marché, dans sa commune natale à laquelle il est indéfectiblement lié22. Si effectivement les rigueurs de la vie militaire ont façonné de nouveaux comportements et ont contraint de nombreux officiers à une mobilité plus active que l'ensemble de la population française, le dernier exemple montre que les réseaux sociaux et familiaux contribuent à un solide enracinement géographique ${ }^{23}$. En revanche lorsque ces liens 
sont rompus, c'est le début d'une vie itinérante qui ne semble pas être souhaitée au préalable par les intéressés. La guerre a, de ce fait, perturbé un certain nombre de repères mais, par la découverte de nouveaux horizons, elle a permis à ces hommes de s'ouvrir au monde rendant plus facile, lorsque les conditions sociales l'exigent, l'opportunité de migrer. Habitués au choc culturel et aux découvertes de nouveaux modes de vie, certains n'hésitent pas à découvrir de nouveaux territoires. Le fait de ne pas retrouver le destin de plus de 78 d'entre eux montre en effet que les mobilités sont beaucoup plus importantes que ne le laissent entendre les documents. Toutefois, sédentarisés ou nomades, les anciens officiers de la Grande Armée, malgré leurs nombreuses blessures, jouissent d'une longévité qui les distingue, une nouvelle fois, de leurs contemporains.

La vieillesse des anciens officiers de la Grande Armée

11 Alors que l'on dénombre 44 décès entre 1815 et 1830, on en recense 71 sur les tables de succession de la décennie 1830,79 pour la suivante, 75 pour celle de 1850,35 pour celle de 1860 puis 10 entre 1871 et 1880 et enfin 3 après $1881.45 \%$ des vétérans meurent entre 1841 et 1859. La Monarchie de Juillet est le régime politique qui voit le plus grand nombre de disparitions avec un total de 137. L'âge moyen au décès est de 72,3 ans alors que sur le plan national il est de 40 ans en $1850^{24}$. De plus la longévité des officiers semble un peu plus importante que celle des sans-grade estimée à 69 ans et demi ${ }^{25}$. Si les décès s'étalent aussi loin dans le temps, puisque les derniers sont enregistrés sous la III ${ }^{\text {e }}$ République, c'est tout d'abord parce que les officiers de la Grande Armée appartiennent à plusieurs générations. Il faut se rappeler qu'en 1814 cohabitent au sein des mêmes unités des anciens, partis en 1792-1793, souvent capitaines ou chefs de bataillons qui ont obtenu leurs épaulettes entre Valmy et Wagram, et de jeunes souslieutenants et lieutenants à peine sortis de l'école spéciale militaire de Saint-Cyr, nés dans les premiers mois de la République ou sous la Terreur, bercés par les victoires impériales durant leur jeunesse et qui ne connaissent, en tant qu'acteurs, que les désastres de la fin du règne. Entre ces deux âges "extrêmes", on remarque une multitude de conscrits ou d'engagés volontaires partis à 20 ans entre 1798 et 1812 qui ont connu les années de gloire puis les années difficiles. Les mentalités, forgées aussi par les origines sociales, le niveau d'instruction et l'expérience de chacun, n'ont guère renforcé au fil du temps la cohésion d'un groupe qui n'a en commun que le respect pour le souverain qu'il a servi.

12 Il ne faut pas croire cependant que les anciens officiers charentais de la Grande Armée atteignent tous l'âge de la vieillesse. L'âge moyen au décès varie en effet selon les décennies. Alors qu'il est plus ou moins élevé sous la Monarchie de Juillet (65 ans) et très élevé sous le Second Empire (76 ans), il n'est que de 54 ans entre 1815 et 1830. Parmi les 44 décès enregistrés au cours de cette période, on dénombre un grand nombre de trentenaires ou de quadragénaires. Nous ne connaissons pas malheureusement la cause du décès pour l'ensemble de cet échantillon mais l'étude des dossiers de retraite montre qu'il s'agit souvent d'officiers ayant reçu des blessures très graves ou qui ont "du mal à se relever des fatigues de la guerre ", expression assez vague utilisée par certains officiers de santé lors de la dernière revue d'inspection dans les années 1815-1816. S'agit-il de suicides liés à des fins d'existence difficiles et solitaires? Nulle source ne nous permet d'y répondre clairement en raison du tabou qui entoure cette fin tragique dans la France du XIX ${ }^{\mathrm{e}}$ siècle ${ }^{26}$. Un cas néanmoins a été identifié. Il concerne le jeune lieutenant Gédéon de Chamborant « atteint d'une maladie mentale incurable qui proviendrait du froid éprouvé en Russie ». Le docteur Esquirol, 
qui considère les suicidés comme des aliénés mentaux, se contente d'observer ce cas atypique qu'il faut peut-être considérer comme un traumatisme de guerre, maladie qui ne sera reconnue par la médecine militaire qu'à l'issue de la première guerre mondiale ${ }^{27}$. Les rapports qui se succèdent chaque année, insistent sur sa «misanthropie» et sa paranoïa qui s'aggravent de mois en mois, sa phobie de la couleur blanche que les médecins mettent en relation avec la neige de Russie. Après plusieurs tentatives infructueuses, cet ancien saint-cyrien se jette dans le vide alors qu'il est interné à l'hôpital de Charenton, le 25 août 1824. Il meurt ainsi à l'âge de 38 $a^{28}{ }^{28}$. L'autre motif de décès qui peut expliquer une faible longévité, est le duel qui entraîne au moins le décès de deux jeunes officiers charentais employés, sous la Restauration, dans des régiments d'infanterie. Leur manque d'expérience mais surtout l'attachement à l'honneur, dans cette société d'après-guerre en proie à la violence individuelle issue de l'Ancien Régime, peuvent expliquer ces disparitions précoces qui touchent particulièrement les villes de garnison et les militaires ${ }^{29}$.

Pour les périodes suivantes, il semble en revanche que ce sont les maladies dues à la vieillesse qui emportent dans la tombe les vétérans de l'Empire ${ }^{30}$. Certaines nécrologies, paraissant dans les journaux locaux, font état de «mort brutale » ou d' « apoplexie » ce qui ne veut pratiquement rien dire ${ }^{31}$. Les testaments olographes rédigés par les mourants laissent quelques indices. On parle volontiers de «longue maladie » ou de "mal incurable $"^{32}$. Des inventaires après décès sont plus éloquents et évoquent la " démence sénile » de certains ${ }^{33}$. Il est très difficile de dire si les blessures de guerre ont pu entraîner la mort, vingt ou trente ans après, d'officiers assez gravement touchés. Néanmoins, sur 329 individus, on sait, d'après les états de service, qu'un peu moins de la moitié a été blessée sous l'Empire. Un rapport rédigé par la mairie d'Angoulême, en 1831 , comptabilise 18 « invalides » pour blessure de guerre sur un total de 43 vétérans ${ }^{34}$. Ne serait-il pas légitime de penser, pour certains cas, à une détérioration de l'état de santé due à des complications résultant de blessures mal cicatrisées? On peut ainsi évoquer l'exemple du lieutenant-colonel Limouzain qui a eu la jambe droite fracassée par un boulet à Hanau. Les autorités municipales et militaires locales le déclarent plusieurs fois «boiteux » dans leurs différents rapports d'inspection. Mais en 1850 un cliché photographique, conservé soigneusement par ses descendants, le représente avec sa jambe droite amputée ${ }^{35}$. S'agit-il d'une complication de cette blessure de guerre qui a rappelé quotidiennement à la victime sa jeunesse passée sur les champs de bataille? Disposant d'une documentation très lacunaire à ce sujet, il paraît impossible d'établir des statistiques d'ensemble ni de conclure d'une manière précise et générale. La mise en relation de sources privées et publiques permet à peine d'éclairer quelques cas individuels. Cependant il est certain que la guerre, malgré les honneurs et la gloire qui ont contribué à une tardive héroïsation, a profondément changé l'existence de ces hommes que l'historiographie traditionnelle s'acharne à faire passer pour des nostalgiques de l'Empire alors que celui-ci a induit pour eux de grandes souffrances. Or ce sont les épaulettes dorées ou argentées et ces cicatrices douloureuses que certains utilisent volontairement pour influencer le regard de leurs contemporains qui les identifient au fil du temps à de " valeureux guerriers » de l'« ancienne armée $»^{36}$.

Mort et identité

14 Ainsi lorsque leur décès est enregistré à la mairie ou consigné sur les tables de succession, on les considère avant tout comme d'« anciens militaires» ou comme des " officiers retraités ». Dans la plupart des cas on met en valeur le grade qui est toujours mentionné pour le capitaine, les officiers supérieurs et généraux. Les fonctions de sous- 
lieutenant ou de lieutenant, considérées par les notables comme des fonctions trop subalternes et moins glorieuses, sont moins fréquemment évoquées par les employés de l'état-civil ou par toute autre autorité. Ce sont parfois les familles qui viennent faire la déclaration et qui se contentent de la formule générale évoquée plus haut. En revanche, si l'officier a été fait chevalier ou officier de la Légion d'honneur, cette distinction est toujours mentionnée dans les actes, preuve de son importance pour la famille du défunt et pour les autorités municipales. Dans $11 \%$ des cas elle efface, parfois, le grade qui est relégué au second plan. Il existe, par ailleurs, des hommes dont le passé militaire est, malgré eux, totalement oublié au moment de l'enregistrement de leur décès. N'ayant pas eu la chance de jouir d'une pension militaire, ils se reconvertissent à leur retour dans divers métiers ou reprennent celui qu'ils ont abandonné au moment de leur incorporation. Le cordonnier Michel Garive, qui compte pourtant dix ans de service dont deux avec le grade de sous-lieutenant sans avoir été blessé, meurt en 1856 en étant identifié comme " maître cordonnier ", condition qu'il a été obligé d'embrasser, selon sa propre déclaration, à son retour en $1814^{37}$. Ce sont aussi des officiers qui ont quitté volontairement l'armée pour reprendre l'entreprise familiale, tels les frères Turner, anciens saint-cyriens, ayant effectué plus de 5 ans de campagne, qui sont désignés comme des "propriétaires ${ }^{38}$. Parfois des proches sont tentés d'insister sur le passé militaire. Marc Laroche, décédé en 1857 à Montmoreau, est considéré comme un "débitant de tabac» mais également comme un «ancien officier $"^{39}$. S'agit-il des dernières volontés du défunt ? N'avons-nous pas affaire à des individus partagés entre deux identités sociales? Il faut rappeler, à ce sujet, que la période 1815-1818 est capitale pour ces officiers qui ont combattu dans l'armée française, pour la plupart, pendant de nombreuses années. Un très grand nombre est mis à la retraite ce qui n'altère en rien leur qualité de militaire et de surcroît d'officier. En revanche le moment est plus difficile pour ces conscrits qui, gagnant l'épaulette par leur instruction, se retrouvent sous-lieutenant ou lieutenant après le désastre russe. Or ils ne comptabilisent pas assez d'années de service pour que le gouvernement royaliste leur accorde une pension. Les bouleversements politiques constituent pour cette catégorie une rupture majeure qui interrompt un processus d'identification entamé sous l'Empire. Ce problème ne se pose pas en revanche pour les fils de notables devenus officiers sous l'Empire et qui, malgré l'obtention d'une pension, acceptent volontiers d'embrasser de nouvelles carrières honorifiques. Les grades obtenus lors des campagnes s'effacent largement dès lors qu'on est devenu maire d'une grande ville, sous-préfet ou riche négociant. Tous les maires d'Angoulême ayant fait une brillante carrière militaire sous l'Empire (quatre sur dix de 1815 à 1855), sont salués, au moment de leur décès, en tant qu'ancien premier magistrat de la ville. Il est rarement fait état de leur grade et de leur passé dans l'armée ${ }^{40}$.

15 L'enregistrement de la mort à la mairie est aussi l'occasion de mesurer les fragiles solidarités qui existent entre les vétérans de l'Empire et plus particulièrement les officiers ${ }^{41}$. Le compagnon d'armes est très rarement le témoin du décès. Il s'agit surtout de membres de la famille, de voisins ou d'employés municipaux dans les bourgs assez importants. Il faut bien se garder cependant de conclusions trop hâtives et il faut, une fois encore, faire appel à une source privée qui s'est révélée extrêmement importante à ce sujet. Dans le récit qu'il fait de son enfance, Auguste Brothier, fils du sous-lieutenant que l'on a déjà cité, évoque les nombreuses visites que son père reçoit de la part de camarades ayant partagé les mêmes souffrances que lui à la guerre. Le capitaine Charles Pichon, souvent imbibé d'«alcool», vit pendant six ans dans l'une des 
chambres de Louis Brothier. La plupart sont originaires de la même commune ou du même canton. Un seul provient d'une localité éloignée de 24 kilomètres, mais la distance n'est pas un obstacle pour ces hommes qui passent tous les dimanches ensemble. En examinant de très près les carrières de ces hommes, on se rend compte qu'ils ont tous servi dans la même compagnie de 1791 à 1802. Beaucoup font partie des mêmes réseaux de parentèle mais d'autres y sont complètement étrangers et il est indéniable que les dangers et les souffrances partagés ont renforcé des liens préalablement constitués. Auguste Brothier évoque même les cotisations organisées au sein de ce petit groupe au bénéfice de l'un d'entre eux, mort sans aucune ressource. La somme récoltée permet alors d'organiser des obsèques décentes pour cet ancien militaire qui semble avoir été oublié par la société civile ${ }^{42}$.

Les nombreux documents concernant les funérailles et la police des cimetières de la commune d'Angoulême permettent de reconstituer en partie le déroulement des obsèques des anciens officiers de l'Empire. Selon un règlement rédigé en avril 1808 et qui n'est guère modifié par la suite, les anciens officiers doivent être enterrés selon un code très strict qui démontre l'importance que leur accorde l'Empire mais également le régime des notables du XIX ${ }^{e}$ siècle. Le règlement prévoit, en effet, à Angoulême, quatre types de funérailles variables selon la catégorie sociale à laquelle le défunt appartient et qui correspondent au schéma mental des autorités de la ville. Selon un texte de 1834, qui reprend les décisions de 1808, les funérailles de première classe coûtent 24 francs et se composent d'un corbillard à deux chevaux avec drap mortuaire garni de franges d'argent. Il faut dépenser 12 francs pour financer un enterrement de deuxième classe avec un corbillard tiré par un cheval avec drap mortuaire garni de soie. Quant aux funérailles de troisième et quatrième classe, elles coûtent respectivement 3 et 6 francs pour un corbillard à un cheval garni en laine ou sans ornement. Il est clairement stipulé que les officiers retraités font partie de la première classe. En outre, on exige pour cette dernière catégorie la présence d'une compagnie d'infanterie commandée par un capitaine qui doit accompagner le corps depuis l'église jusqu'au cimetière ${ }^{43}$. Cette discrimination flagrante et codifiée n'empêche pas, toutefois, les conflits politiques entre la mairie et l'opposition, notamment à l'annonce de la mort d'anciens officiers de la Grande Armée. Sous la Restauration, le préfet de la Charente est très embarrassé lorsqu'il apprend le décès du chef de bataillon Jean Thouars, en janvier 1830, qui « au moment suprême aurait repoussé les secours de la religion ». L'aumônier de l'hôpital ainsi que le maire d'Angoulême, le baron Chasteignier, ancien officier de l'Empire, s'appuient sur les "principes qui étaient d'avis que le défunt n'avait aucun droit aux prières et aux cérémonies extérieures ». De plus l'indigence, la malhonnêteté et les opinions républicaines, trop connues de tous les notables d'Angoulême, ne servent pas la cause du défunt. Cependant le préfet et l'évêque sont obligés, d'un commun accord, de ramener tout le monde à la raison. Le premier craint surtout, dans la lettre qu'il adresse le 29 janvier 1830 au ministre de l'Intérieur, les " pamphlets de l'opposition » et un "tumulte» inutile dans la ville ${ }^{44}$. Le maire d'Angoulême prend alors toutes les mesures qui s'imposent et annonce au commandant de la place des funérailles militaires de première classe pour le chef de bataillon Thouars. Les officiers du 36e Régiment d'Infanterie, alors en garnison à Angoulême, dont une compagnie participe aux obsèques, se cotisent pour "les frais de l'enterrement » de ce commandant mort "sans laisser la plus légère somme " $^{45}$. Le maire et le préfet du Second Empire sont confrontés à un problème à peu près similaire lorsqu'est enterré, en 1851, le chef d'escadron Jean Horric de la Motte de Beaucaire, mort à Trieste en Italie alors qu'il 
vient de rendre visite au comte de Chambord en exil. Ancien officier de Napoléon Ier, cet homme qui appartient à l'une des plus vieilles familles nobles de la Charente, n'a jamais caché ses sentiments royalistes et ce n'est pas un drapeau tricolore que la famille veut sur le cercueil du défunt mais un drapeau blanc frappé de la fleur de lys. Les autorités sont très embarrassées et il faut toute la diplomatie du maire d'Angoulême, lui aussi ancien officier de cavalerie de l'Empire comme le défunt, pour éviter le scandale. La dépouille de Jean Horric de la Motte de Beaucaire est finalement drapée d'une oriflamme blanche tout en recevant les honneurs militaires dûs à son grade ${ }^{46}$.

17 Cependant, ce règlement strict masque une réalité beaucoup plus discriminatoire qui démontre à quel point l'origine sociale d'un officier de la Grande Armée revêt d'importance pour les élites locales angoumoisines. Il faut appartenir à une famille reconnue de la ville pour avoir droit, effectivement, à une nécrologie dans les différents journaux locaux, qu'ils soient de tendance royaliste, libérale ou républicaine. Sur 44 officiers décédés au chef-lieu du département entre 1830 et 1877, 14 seulement ont droit à un hommage solennel dans la presse qui salue toujours la disparition d'un "valeureux guerrier ». S'il s'agit d'un membre de la garde nationale, le convoi funèbre est bercé par les "sons voilés des tambours ", la " croix d'honneur et les épaulettes " décorent le cercueil de ce vétéran qui a su «noblement gagner son grade sur les champs de bataille». L'éloge ne se borne pas cependant à la carrière militaire et on évoque volontiers sa "vie civile», la "dignité de sa conduite » qui a suscité «l'estime de ses concitoyens ». Les obsèques sont alors l'occasion, pour l'homme politique local, de récupérer à son profit l'image de ces guerriers. Lors de l'enterrement du capitaine Joseph Poitevin à Confolens, le futur député républicain Laribière prononce un discours devant la foule dans lequel, après avoir évoqué la "gloire ", la "bravoure " et l'honneur de l'un des " débris de la république et de l'empire [...] mutilés par le fer ", il célèbre à maintes reprises le "peuple » et certains acquis de la révolution de 1789 comme l'égalité civile. Il dénonce également les "absurdes haines nationales" fomentées par les «tyrans et les monarques ${ }^{47}$. N'hésitant pas à faire l'amalgame entre l'armée de la Révolution et celle de l'Empire, Babaud-Laribière célèbre ainsi leur mémoire en faisant retomber les responsabilités de la guerre sur la monarchie.

Dans une société qui voit le retour en force des pratiques religieuses dans les milieux aisés, on gomme en revanche les faits jugés immoraux par la notabilité locale que l'on peut entrevoir dans les états de service et dans les inventaires après décès. Ainsi, la nécrologie du lieutenant-colonel Limouzain, qui occupe une très large place dans la troisième feuille du journal Le Charentais du 24 mars 1856, vante l'exemplarité de ce personnage, père de trois enfants et marié avec la fille d'un avocat ${ }^{48}$. Le journaliste oublie volontairement de mentionner la vie conjugale plutôt dissolue de ce «brave guerrier » qui, en réalité, n'a pas cherché à cacher lors de son retour les deux enfants qu'il avait honnêtement reconnus lors de son séjour à Corfou entre 1806 et 1813, fruits d'une union avec une Italo-Grecque aux origines obscures ${ }^{49}$. Les lignes qui lui sont consacrées n'évoquent nullement tout le soin que cet officier supérieur a développé pour donner une solide éducation à ses deux enfants qui ne sont pas oubliés dans le testament qu'il rédige un an avant sa mort ${ }^{50}$. Ceci rend bien compte des qualités que l'élite attend d'un officier de l'armée française. Brave et courageux, ce dernier doit aussi respecter un code moral qui est loin de faire l'unanimité parmi les vétérans mais qui est précisément celui de la «bonne société ». 
19 C'est pourtant l'honneur et la gloire que ces derniers ou leur famille tentent de mettre en valeur devant l'éternité. La recherche des tombes dans les cimetières de Charente et la lecture des registres des cimetières apportent des informations capitales sur le regard que posent les proches et parfois l'officier lui-même sur sa propre condition. Parmi les 242 officiers morts dans le département, ont été retrouvées jusqu'à maintenant 32 tombes plus ou moins bien conservées ${ }^{51}$. Ce sont pour la plupart des sarcophages ou des stèles funéraires verticales surmontées d'une amphore. Ce dernier modèle, ainsi que les colonnes et les obélisques, font leur apparition vers 1840-1850. Les tombes des anciens officiers sont conformes à la mode funéraire chère aux notables de la Monarchie de Juillet et du Second Empire ${ }^{52}$. Cependant, malgré cet attrait pour le néo-classicisme, les vétérans de l'Empire semblent de nouveau, dans l'au-delà, se distinguer de leurs contemporains en affirmant de manière très nette leur identité militaire ${ }^{53}$. Les épitaphes qui ornent les stèles funéraires et qui ont été soigneusement préparées par la famille du défunt ou par l'officier - il est difficile de le savoir car les testaments ne font pas mention de dernières volontés qui sans nul doute sont transmises oralement -, s'attardent sur son grade et son statut ${ }^{54}$. On peut ainsi lire sur la tombe d'Arnaud Pallet sa qualité de « lieutenant retraité ». L'observation des autres tombes montre que les militaires, les médecins et les prêtres sont les seules catégories à mettre autant en valeur leur qualité professionnelle. Si l'officier a fait preuve de bravoure, la Légion d'honneur ou la croix de Saint-Louis sont non seulement écrites en toutes lettres mais l'insigne est sculpté sur la pierre tombale. Les familles font parfois état des campagnes ou des batailles. Plus le grade du défunt est élevé, plus la tombe est ornée de référents militaires spécifiques. On fait ainsi sculpter un shako, des épaulettes, une épée et des drapeaux. Jamais en revanche il n'est fait mention de l'empereur, y compris pour les officiers morts sous le Second Empire. Cela confirme le fait que ces hommes se considèrent avant tout comme des militaires serviteurs de l'État, préfigurant ainsi l'armée française du $\mathrm{XX}^{\mathrm{e}}$ siècle ${ }^{55}$. Mais cette extériorisation de l'identité, au moment suprême, montre que l'armée n'est pas cette institution qui a perdu son prestige après Waterloo comme l'ont affirmé parfois certains historiens ${ }^{56}$. Le cimetière romantique du $\mathrm{XIX}^{\mathrm{e}}$ siècle, devenu lieu de promenade pour une part croissante de Français, est l'ultime moyen de montrer son rang parmi la société des notables ${ }^{57}$. N'est-ce pas aussi un vecteur par lequel l'officier et sa famille veulent lutter contre l'oubli? La tombe, pour ceux qui inscrivent leurs campagnes, n'est-elle pas devenue un lieu de mémoire de la guerre? Des modèles de plaque semblent exister pour les militaires. Deux tombes du même type ont été retrouvées dans un rayon de cinq kilomètres. Après avoir présenté les qualités du défunt, on précise qu'il a participé aux combats de " 1792 jusqu'en 1815 ». Suivent alors les armées dans lesquelles il a servi. Le tout est surmonté de drapeaux, de l'inscription « Honneur et Patrie » tandis que deux croix de Légion d'honneur sont disposées latéralement. En s'adressant de la sorte aux civils qui errent dans les cimetières, dont les allées ont été élargies pour faciliter la marche des passants, la famille du défunt entend perpétuer le souvenir du vétéran qui a combattu au service d'une nation qui se doit d'être reconnaissante.

Cependant les officiers sont nombreux à avoir pris certaines dispositions avant leur mort. Lorsque le maire d'Angoulême, en 1835, autorise l'achat de concessions funéraires perpétuelles ou trentenaires, ils sont nombreux à remplir le dossier d'acquisition. Parmi les dix premières concessions ouvertes figurent trois officiers. Sur les 34 morts à Angoulême, 20 agissent de la sorte entre 1835 et 1877. Les dossiers, conservés aux archives municipales d'Angoulême, démontrent que l'acheteur désire à 
chaque fois « conserver les restes » d'un être cher ${ }^{58}$. Ces hommes s'inscrivent bien dans le XIX ${ }^{e}$ siècle qui s'ouvre au "culte des morts " ${ }^{59}$. À défaut de les considérer comme les initiateurs exclusifs de cette révolution culturelle, on peut légitimement les percevoir comme les acteurs majeurs l'ayant accélérée ${ }^{60}$. Ne faut-il pas y voir alors les traces indélébiles des guerres napoléoniennes où les corps, les soirs de bataille, sont jetés pêle-mêle dans de vastes fosses communes ? Est-ce la vision de ces corps enchevêtrés et sans sépulture décente qui conditionne ces nouveaux comportements face à la mort ?

Quelle que soit l'origine sociale des officiers de la Grande Armée, l'expérience de la guerre a été un facteur déterminant qui a modelé en profondeur leurs attitudes et leurs comportements depuis la fin de l'Empire jusqu'au décès du dernier d'entre eux. Elle apparaît comme un épisode majeur de leur vie dont le souvenir est matérialisé par la conservation d'objets militaires spécifiques révélés à l'occasion des inventaires après décès. Sur les 32 retrouvés et exploités, l'épée et les épaulettes, symboles du commandement et du rang, apparaissent trente fois. Sont désignés par la suite dans des proportions beaucoup plus faibles, les coiffures (shako ou bicorne) et quelques armes à feu (pistolets et fusils). L'uniforme dans son intégralité n'est mentionné que lorsque le défunt a servi dans une unité prestigieuse comme la garde impériale ${ }^{61}$. Cependant, tous ces "souvenirs" ne sont conservés que fort discrètement, dans l'armoire ou dans le placard de la chambre du défunt, c'est-à-dire dans le lieu le plus intime du domicile. Aucun vétéran n'a affiché avec ostentation son passé militaire : aucun effet n'est exposé dans le salon ou la salle à manger. De plus, la très faible part des ouvrages relatifs à la guerre et à l'armée dans les bibliothèques privées confirme la forte intériorisation de leur identité militaire dans les dernières années de leur vie. Cette relative discrétion peut expliquer alors la très faible proportion de leurs fils ayant choisi une carrière militaire. Cette dernière ne semble en effet séduire que $18 \%$ de ceux qui sont majeurs au moment du décès de leur père. Cette proportion tombe à $6 \%$ si on retire le contingent des officiers ayant poursuivi leur carrière sous la Restauration et la Monarchie de Juillet. Un examen attentif des registres de conscription de la Charente dans lesquels on peut suivre les réclamations et les motifs d'incorporation montre que les fils d'officiers cherchent dans plus de $70 \%$ des cas à éviter le service militaire. Les engagements volontaires sont rares. La plupart entament en effet une carrière militaire en raison du hasard produit par le tirage au sort ${ }^{62}$. Si la guerre permet aux gradés de se mettre en valeur dans une société pour qui l'honneur revêt une grande importance, il semble aussi qu'elle ait laissé des souvenirs douloureux. La vision de milliers de morts sur un champ de bataille, même glorieux, ainsi que le cri des blessés agonisants, ont sans doute développé chez certains un sentiment pacifiste susceptible de décourager les éventuelles vocations militaires de leurs enfants.

Alors que l'historiographie classique, victime du regard porté par les artistes du XIX siècle, a forgé un seul et même visage du vétéran de l'Empire, cette étude permet au contraire de redessiner le portrait de ces hommes à l'aune des nouvelles problématiques propres à l'histoire sociale et culturelle. Loin d'être un groupe homogène, fidèle à son souverain, les officiers de la Grande Armée présentent toutes les caractéristiques d'une catégorie qui n'a de commun que l'expérience guerrière vécue sur les champs de bataille européens. Le regard instantané posé au crépuscule de leur vie a permis de saisir la variété des situations au cours de ces années d'après-guerre, souvent longues, parfois douloureuses et surtout fécondes non seulement pour eux mais aussi pour leurs proches et la société française en général. La guerre, gravée dans les chairs et dans les mémoires, a généré de nouveaux modes de vie et s'il paraît certain 
que la plupart s'accommodent plus ou moins bien des exigences de la notabilité, d'autres finalement luttent pour la reconnaissance d'un rang acquis difficilement et sans enthousiasme. Plus que porteurs de l'aigle impérial, ils sont les messagers discrets et involontaires d'une épreuve remplie de gloires, de désastres et de souffrances à l'origine de multiples ruptures qui ont façonné et recomposé en grande partie la société française.

\section{NOTES}

1.Capitaine CoIGNET, Les cahiers du capitaine Coignet, Paris, Arléa, 2001, 425 p.

2.Louis-François LejeunE, Mémoires du général Lejeune (1792-1814), Paris, ed. Grenadier, 2001, $489 \mathrm{p}$.

3.Octave LeVAVASSEUR, Souvenirs militaires (1800-1815), Paris, Librairie des Deux Empires, 2001, $337 \mathrm{p}$.

4.Capitaine FrançoIs, Journal du capitaine François dit le dromadaire d'Égypte (1792-1830), Paris, Tallandier, 2003, $895 \mathrm{p}$.

5.Honoré de BALZAC, La Comédie humaine, Paris, Gallimard, La Pléiade, 12 volumes, 1976-1981.

6. SHD : les revues de l'an XI et l'enquête de 1814 sont contenues dans la sous-série XB (dossiers administratifs des régiments de l'armée française). En 1816, une commission d'examen, présidée par le Maréchal Victor était chargée d'enquêter sur les officiers qui avaient servi pendant les Cent-Jours. En C16-41 il est possible de lire le rapport du préfet de la Charente. Enfin les registres-matricules des officiers sont compris dans la série 2YB. Il existe en moyenne 5 registres par régiment. N'ont été exploités que les derniers de façon à compléter l'enquête de 1814 qui laissait de côté les officiers faits prisonniers par les troupes coalisées.

7.AD Charente : dans la série $\mathrm{L}$ on peut consulter les listes des bataillons de volontaires confectionnées par les autorités locales alors que la série $\mathrm{R}$ conserve tous les registres de conscription de l'Empire dans lesquels les unités d'incorportation sont mentionnées. Pour obtenir le maximum d'officiers, il convenait ensuite de suivre leur destin dans les registres-matricules des corps de troupe du SHD.

8.SHD : les séries 2, 3 et 4 YF concernent tous les officiers retraités. Quant à la série $2 \mathrm{YE,}$ en cours d'inventaire, elle permet de connaître les officiers démissionnaires ou réformés sans et avec traitement.

9.Guillaume CUCHET, Le crépuscule du purgatoire, Paris, Armand Colin, 2005, p. 23.

10.William SERMAN, Les officiers dans la nation (1848-1914), Paris, Aubier, 1982, p. 145.

11.SHD, 3YF 58001 (dossier de retraite de Jean Saint-Loup).

12.AM Angoulême, recensements de 1822 et 1836 non cotés.

13.Ibidem, copie de lettres de la municipalité (1836-1838) ; document non coté.

14.SHD, 3YF 58001 (dossier de retraite de Jean Saint-Loup).

15.AN, L2774013 (dossier de légion d'honneur du capitaine Jean Saint-Loup).

16.AD Charente, $Q$ paquet 2368 (table de succession du canton de Barbezieux).

17.Ibidem, 1M99 (surveillance des militaires suspects d'opposition au pouvoir royal). 
18.AM Angoulême, copie de lettres (1836-1838); document non coté.

19.AD Charente, Q 2435 (table de successions d'Angoulême).

20.SHD, 3YF 94055 (dossier de retraite du chef de bataillon Jean Thouars).

21.Archives privées de Monsieur Jean-Louis Carde, papiers personnels de Claude

Mathurin Brothier et carnet d'Auguste Brothier son fils.

22.SHD, 2YF 78707 (dossier de retraite du sous-lieutenant Claude Mathurin Brothier).

23.Anne Rolland-Boulestreau, Les notables des Mauges. Communautés rurales et Révolution (1750-1830), Rennes, PUR, 2004, 401 p.

24.Jacques DUPAQUIER, Histoire de la population française, 4 volumes, Paris, PUF, 1995, tome 3 (de 1789 à 1914), p. 6.

25. Natalie PETITEAU, Lendemains d'Empire. Les soldats de Napoléon dans la France du XIX ${ }^{\text {ème }}$ siècle, Paris, La Boutique de l'Histoire, 2003, p. 340.

26.Georges MinoIs, Histoire du suicide. La société occidentale face à la mort volontaire, Paris, Fayard, 1995, p. 369.

27.Louis CROCQ, Les traumatismes psychiques de guerre, Paris, ed. Odile Jacob, 1999, 422

pages

28.SHD, 2YE 653bis (dossier personnel de Gédéon de Chamborant).

29.Pascal BRIOIST, Hervé DRÉVILLON et Pierre SERnA, Croiser le fer. Violences et culture de l'épée dans la France moderne (XVI ${ }^{\text {ème}}$-XVIII ${ }^{\text {ème }}$ siècle), Paris, Champ-Vallon, 2002, p. 468-469. 30.Patrice BOURDELAIS, Le nouvel âge de la vieillesse. Histoire du vieillissement de la population, Paris, Odile Jacob, 1993, 441 p.

31.Georges MiNOIS, op. cit. p. 404.

32.AD Charente, 2 E 9829 (testament du capitaine Giraudeau).

33.Ibidem, 2 E 12333 (inventaire après décès du lieutenant-colonel Limouzain).

34.AM Angoulême, liste des officiers vivants dans la commune d'Angoulême en 1831, non cotée.

35.Archives privées de M. Meslet.

36.AD Charente, nécrologie du capitaine Poitevin dans Le journal de Confolens, 1842.

37.Ibidem, 2 M 149 (secours).

38.Ibidem, état-civil de Saint-Genis d'Hiersac et de Julienne.

39.Ibidem, Q Paquet 1398 (succession de Marc Laroche, canton de Montmoreau).

40.AD Charente : état-civil de Roullet, d'Angoulême et de Bunzac pour les décès de Lambert, Chasteignier, Normand de la Tranchade et Thevet.

41.Pierre Guillaume (dir.), Les solidarités. le lien social dans tous ses états, Colloque de Bordeaux, 16-17 juin 2000, Pessac, Maison des sciences de l'Homme d'Aquitaine, 509 p. 42.Archives privées de M. Jean-Louis Carde ; Anne Rolland-BoulestreAu, Les notables des Mauges. Communautés rurales et Révolution (1750-1830), op. cit., p. 324-335

43.AM Angoulême, I-1 (police du cimetière des Bardines).

44.AN, F7/6768 (situation politique du département de la Charente).

45.AM Angoulême, copie de lettres (1830-1833); document non coté.

46.Ibidem, cahier du secrétaire de mairie Mercier ; document non coté.

47.AD Charente, nécrologie du capitaine Poitevin dans Le journal de Confolens, 1842.

48.Ibidem, Le Charentais du 24 mars 1856.

49.SHD, 2YE (dossier personnel du lieutenant-colonel Limouzain).

50.AD Charente, 2E12584 (testament du lieutenant-colonel Limouzain). 
51.Cimetières des Bardines à Angoulême, de l'Houmeau-Pontouvre, de Champniers, de Peudry, de Vars, d'Availles-Limousine, de Saint-Germain-sur-Vienne, de Balzac, de Brie, de Ruffec, d'Empuré, de Condac, de Chasseneuil, de Taponnat et de La Rochefoucauld. 52.Société d'émulation du Jura (avec la collaboration de Michel BÉDAT, de Roger BERGERET, de Claude-Isabelle BRELOT), Tombes d'autrefois, Lons-le-Saunier, imprimerie Jacques et Demontrond, 1997, 155 p.

53.Jean-Marc FERRER et Philippe GRANDCOING, Des funérailles de porcelaine. De l'art de la plaque funéraire en porcelaine de Limoges au XIX ${ }^{e}$ siècle, Limoges, Culture et Patrimoine en Limousin, 2000.

54.Philippe ARIÈs, Essais sur l'histoire de la mort en Occident. Du Moyen-Âge à nos jours, Paris, Seuil, (1975), p. 52.

55.William SERMAN, op. cit., p. 45.

56. Raoul GIRARDET, La société militaire dans la France contemporaine (1815-1939), Paris, Plon, 1953, $328 \mathrm{p}$.

57. Madeleine LASSÈRE, Villes et cimetières en France de l'Ancien régime à nos jours. Le territoire des morts, Paris, L'Harmattan, 1997, 411 p.

58.AM Angoulême, concessions funéraires (document non coté).

59.Michel Vovelle, La mort et l'Occident de 1300 à nos jours, Paris, Gallimard, 1ère éd.1983, 2ème éd. 2000, 779 p.

60.Guillaume CUCHET, op. cit., p. 23

61.AD Charente, 2 E 10998 (inventaire de François Eutrope Dedé-1832).

62.AD Charente, série $\mathrm{R}$; ont été consultés à cette fin les registres de conscription des classes 1830 à 1850 .

\section{RÉSUMÉS}

Acteurs majeurs de l'Empire, les officiers de la Grande Armée, dont les témoignages célèbres et précieux ont servi de base à de nombreux travaux, constituent un groupe dont l'image a été entièrement façonnée par la légende napoléonienne. Pourtant l'étude de leurs fins de vie montre qu'il s'agit d'une population dont l'existence et les trajectoires d'après-guerre, loin de correspondre aux clichés véhiculés par la littérature et la peinture du XIX ${ }^{\mathrm{e}}$, ont été profondément marquées par l'expérience de la guerre, laquelle se manifeste par un ensemble de comportements et d'attitudes qui contrastent parfois avec les pratiques culturelles et sociales de la population française de la seconde moitié du XIX ${ }^{\mathrm{e}}$ siècle. Par l'intérêt porté à la question des représentations et de l'identité, cet article se propose d'accorder une place importante aux derniers gestes de ces hommes et à l'image qu'ils ont bien voulu laisser d'eux-mêmes à travers le regard de leurs concitoyens.

The death of the former officers of the Grande Armée: the example of the Charentais. Major participants in the Empire, the officers of the Grande Armée whose testimony, celebrated and precious, has served as the basis of numerous works constitute a group whose image was entirely shaped by Napoleonic legend. But a study of the end of their lives shows that their lives after the war, far from corresponding to the cliches spread by literature and painting of the 19th century, had been deeply marked by the experience of war characterized by certain behavior and 
attitudes that sharply contrasted at times with the cultural and social practices of the rest of the French population in the second half of the nineteenth century. This article informed by the question of representation and identity analyzes the final acts of these men as well as the image they left of themselves in the eyes of their fellow citizens.

INDEX

Mots-clés : officiers, honneur, Grande Armée, mobilité, réinsertion, rang, identité, mort, expériences de guerre

\section{AUTEUR}

\section{STÉPHANE CALVET}

Doctorant à l'Université d'Avignon

UFR Lettres et sciences humaines

74 rue Louis Pasteur

84029 Avignon Cedex

Stephane.Calvet@wanadoo.fr 